\title{
The scalar radius of the pion from lattice QCD in the continuum limit
}

\author{
Vera Gülpers $^{1, a}$, Georg von Hippel ${ }^{1}$, and Hartmut Wittig ${ }^{1,2}$ \\ 1 PRISMA Cluster of Excellence and Institut für Kernphysik, Johannes Gutenberg-Universität Mainz, 55099 Mainz, Germany \\ 2 Helmholtz Institute Mainz, Johannes Gutenberg-Universität Mainz, 55099 Mainz, Germany
}

Received: 20 August 2015 / Revised: 10 November 2015

Published online: 9 December 2015

(C) The Author(s) 2015. This article is published with open access at Springerlink.com

Communicated by R. Alkofer

\begin{abstract}
We extend our study (Phys. Rev. D 89, 094503 (2014)) of the pion scalar radius in two-flavour lattice QCD to include two additional lattice spacings as well as lighter pion masses, enabling us to perform a combined chiral and continuum extrapolation. We find discretisation artefacts to be small for the radius, and confirm the importance of the disconnected diagrams in reproducing the correct chiral behaviour. Our final result for the scalar radius of the pion at the physical point is $\left\langle r^{2}\right\rangle_{\mathrm{s}}^{\pi}=0.600 \pm 0.052 \mathrm{fm}^{2}$, corresponding to a value of $\bar{\ell}_{4}=4.54 \pm 0.30$ for the low-energy constant $\bar{\ell}_{4}$ of NLO chiral perturbation theory.
\end{abstract}

\section{Introduction}

Hadronic form factors carry crucial information about the internal structure of hadrons as bound states of quarks and gluons in Quantum Chromodynamics (QCD). Since the dynamics underlying this structure is deeply nonperturbative, the only known first-principles approach that allows for the extraction of form factors from QCD with full control of systematic errors is the use of lattice simulations.

The scalar form factor of the pion, defined as

$$
F_{\mathrm{s}}^{\pi}\left(Q^{2}\right) \equiv\left\langle\pi^{+}\left(p_{f}\right)\left|m_{\mathrm{d}} \bar{d} d+m_{\mathrm{u}} \bar{u} u\right| \pi^{+}\left(p_{i}\right)\right\rangle,
$$

with the four-momentum transfer

$$
Q^{2}=-q^{2}=-\left(p_{f}-p_{i}\right)^{2}
$$

is not directly accessible to experiment for lack of a suitable low-energy probe. However, the associated scalar radius,

$$
\left\langle r^{2}\right\rangle_{\mathrm{S}}^{\pi}=-\left.\frac{6}{F_{\mathrm{S}}^{\pi}(0)} \frac{\partial F_{\mathrm{S}}^{\pi}\left(Q^{2}\right)}{\partial Q^{2}}\right|_{Q^{2}=0}
$$

can be related to the experimentally measurable cross section for $\pi \pi$ scattering [1] using chiral perturbation theory $(\chi \mathrm{PT}) . \mathrm{A}$ notable feature of the scalar radius is that in

\footnotetext{
a e-mail: guelpers@kph.uni-mainz.de
}

$\chi \mathrm{PT}$ at NLO it depends only on a single low-energy constant, $\bar{\ell}_{4}$, through $[2,3]$

$$
\left\langle r^{2}\right\rangle_{\mathrm{S}}^{\pi}=-\frac{1}{(4 \pi F)^{2}} \frac{13}{2}+\frac{6}{(4 \pi F)^{2}}\left[\bar{\ell}_{4}+\ln \left(\frac{m_{\pi, \mathrm{phys}}^{2}}{m_{\pi}^{2}}\right)\right],
$$

where the pion decay constant is $F=92.2 \mathrm{MeV}$ [4].

The accurate knowledge of the low-energy constants (see [5] for a recent compilation) is required to enhance the predictive power of chiral effective theory for a number of phenomenological applications.

In lattice QCD, the scalar form factor of the pion is derived from a three-point function which receives contributions from both quark-connected and quark-disconnected diagrams (cf. fig. 1). Due to the large numerical cost disconnected diagrams have often been neglected in lattice calculations. However, arguments based on partially quenched $\chi \mathrm{PT}[6]$ indicate that quark-disconnected contributions to the pion scalar radius could be sizeable. Our earlier work [7], using lattice data at a single value of the lattice spacing and relatively large pion masses, has largely confirmed the prediction of $\chi \mathrm{PT}$. In the present work we extend our study significantly, by including two additional lattice spacings, as well as lower pion masses. This allows for a combined chiral and continuum extrapolation of our results. In this way we obtain improved estimates for the pion scalar radius and the low-energy constant $\bar{\ell}_{4}$, including a full assessment of systematic errors. 


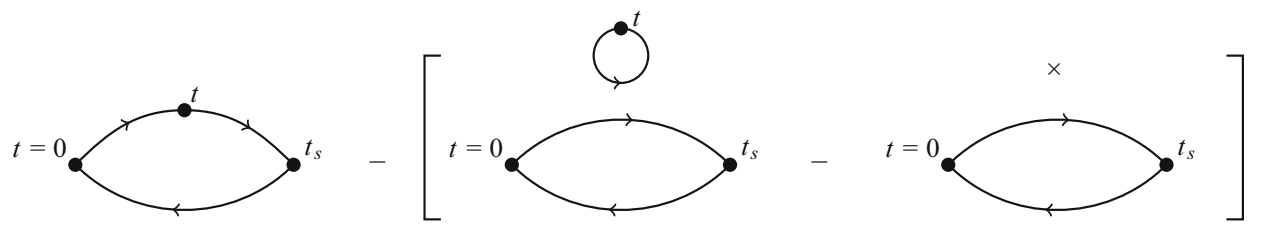

Fig. 1. Quark flow diagrams for the connected and disconnected contributions to the scalar form factor of the pion. The cross represents the vacuum expectation value $\langle\bar{\psi} \psi\rangle$, which for the Wilson fermion discretisation used here contains a power-law divergent additive renormalisation.

Table 1. Overview of the CLS ensembles that have been used for the calculation of the scalar form factor of the pion. The lattice spacing given was determined using the $\Omega$ baryon mass [8]. Note that all ensembles fulfil $m_{\pi} L \geq 4$.

\begin{tabular}{cccccc}
\hline Label & $a[\mathrm{fm}]$ & Lattice & $m_{\pi}[\mathrm{MeV}]$ & $m_{\pi} L$ & $N_{\text {cfg }}$ \\
\hline \hline A3 & 0.079 & $64 \times 32^{3}$ & 473 & 6.1 & 133 \\
A4 & 0.079 & $64 \times 32^{3}$ & 363 & 4.7 & 200 \\
A5 & 0.079 & $64 \times 32^{3}$ & 312 & 4.0 & 250 \\
B6 & 0.079 & $96 \times 48^{3}$ & 267 & 5.1 & 159 \\
\hline E3 & 0.063 & $64 \times 32^{3}$ & 650 & 6.6 & 156 \\
E4 & 0.063 & $64 \times 32^{3}$ & 605 & 6.2 & 162 \\
E5 & 0.063 & $64 \times 32^{3}$ & 456 & 4.7 & 1000 \\
F6 & 0.063 & $96 \times 48^{3}$ & 325 & 5.0 & 300 \\
F7 & 0.063 & $96 \times 48^{3}$ & 277 & 4.3 & 351 \\
G8 & 0.063 & $128 \times 64^{3}$ & 193 & 4.0 & 348 \\
\hline N5 & 0.050 & $96 \times 48^{3}$ & 430 & 5.2 & 477 \\
N6 & 0.050 & $96 \times 48^{3}$ & 332 & 4.1 & 946 \\
O7 & 0.050 & $128 \times 64^{3}$ & 261 & 4.2 & 490 \\
\hline
\end{tabular}

\section{Methods and results}

\subsection{Ensembles used}

In our calculation of the scalar radius we use $N_{f}=2$ dynamical flavours of non-perturbatively $O(a)$-improved Wilson fermions. The gauge ensembles have been generated by the CLS initiative and are listed in table 1 .

Compared to our previous work [7] we have included two more lattice spacings, a coarser one, $a=0.079 \mathrm{fm}$ (labels $\mathrm{A}$ and $\mathrm{B}$ ), and a finer one, $a=0.050 \mathrm{fm}$ (labels $\mathrm{N}$ and $\mathrm{O})$. For the intermediate lattice spacing, $a=0.063 \mathrm{fm}$, the G8 ensemble with a pion mass of $193 \mathrm{MeV}$ has been included in our calculation. The use of different lattice spacings allows for performing a continuum extrapolation of our results for the scalar radius besides the chiral extrapolation. In general one also has to extrapolate to infinite volume, however, all our ensembles fulfil $m_{\pi} L \geq 4$ and thus we expect finite volume effects to be negligible.

\subsection{Form factor calculation}

We explicitly calculate the connected and the disconnected contribution to the matrix element $\langle\pi|\bar{q} q| \pi\rangle$ for three different momentum transfers and determine the scalar pion form factor. The three momentum transfers are chosen such that the pion has momentum $\left|\mathbf{p}_{i}\right|=0$ at the source and momentum $\left|\mathbf{p}_{f}\right|=0,\left|\mathbf{p}_{f}\right|=2 \pi / L$ or $\left|\mathbf{p}_{f}\right|=\sqrt{2} 2 \pi / L$ at the sink.

The quark loop required for the disconnected part is estimated using three stochastic sources per timeslice $t$ and a generalised Hopping Parameter Expansion to 6th order. Further details can be found in [7].

We use ratios [9] of three- and two-point functions to extract the desired scalar matrix element from our data. For the connected contribution we use

$$
R_{1}\left(t, t_{s}, \mathbf{p}_{i}, \mathbf{p}_{f}\right)=\sqrt{\frac{C_{3}\left(t, t_{s}, \mathbf{p}_{i}, \mathbf{p}_{f}\right) C_{3}\left(t, t_{s}, \mathbf{p}_{f}, \mathbf{p}_{i}\right)}{C_{2}\left(t_{s}, \mathbf{p}_{i}\right) C_{2}\left(t_{s}, \mathbf{p}_{f}\right)}},
$$

where the pion sink is located on $t_{s}$ and the scalar operator is inserted at time $t$. The pion source is placed at $t=0$ for simplicity. The required two-point and connected three-point functions have been calculated with Gaussian smearing [10-12] applied to the source.

For the disconnected contribution we use smearedsmeared two-point correlation functions and the ratio

$$
\begin{aligned}
R_{3}\left(t, t_{s}, \mathbf{p}_{i}, \mathbf{p}_{f}\right)= & \frac{C_{3}\left(t, t_{s}, \mathbf{p}_{i}, \mathbf{p}_{f}\right)}{C_{2}\left(t_{s}, \mathbf{p}_{f}\right)} \\
& \times \sqrt{\frac{C_{2}\left(t_{s}, \mathbf{p}_{f}\right) C_{2}\left(t, \mathbf{p}_{f}\right) C_{2}\left(\left(t_{s}-t\right), \mathbf{p}_{i}\right)}{C_{2}\left(t_{s}, \mathbf{p}_{i}\right) C_{2}\left(t, \mathbf{p}_{i}\right) C_{2}\left(\left(t_{s}-t\right), \mathbf{p}_{f}\right)}}
\end{aligned}
$$

To increase the statistics for the disconnected contribution we average over four different pion source positions.

In our previous work [7] we have discussed the remaining time-dependences of these ratios due to periodic boundary conditions and the backward propagating pion in the two-point function. We take these dependences into account by dividing the ratios $R_{1}$ and $R_{3}$ by the appropriate time-dependent factors. Furthermore, we have shown significant excited state contributions for small source-sink separations of $t_{s}<1.5 \mathrm{fm}$. This has been confirmed on our extended set of ensembles. Thus, for all ensembles we only use data with $t_{s}>1.5 \mathrm{fm}$. Further details on the extraction of the scalar form factor can be found in [7].

For all ensembles we observe a significant contribution of the disconnected diagram to the scalar form factor. However, we find the relative disconnected contribution to the form factor to be strongly dependent on the 


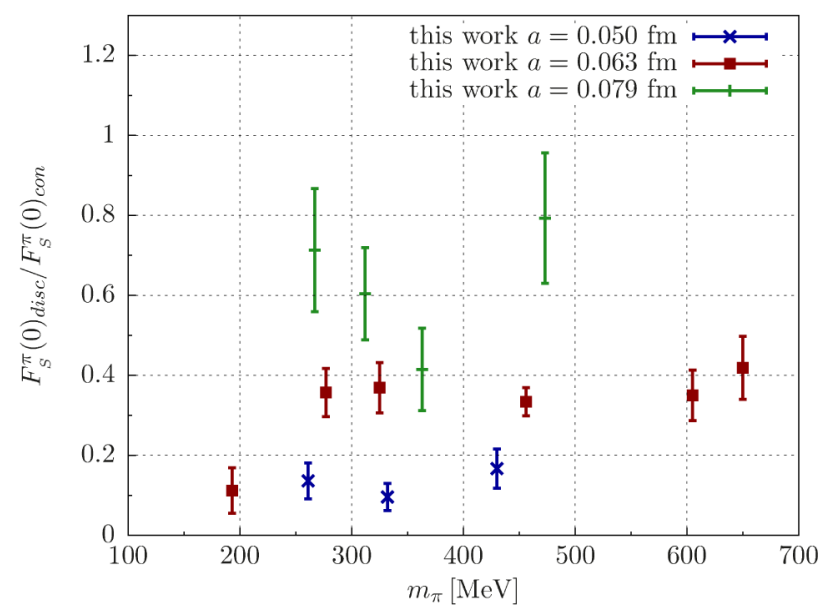

Fig. 2. The disconnected contribution $F_{\mathrm{S}}^{\pi}(0)_{\text {disc }}$ at $Q^{2}=0$ divided by the corresponding connected contribution. Blue, red and green points denote different values of the lattice spacing $a=0.050 \mathrm{fm}, 0.063 \mathrm{fm}$ and $0.079 \mathrm{fm}$, respectively.

lattice spacing. Figure 2 shows the disconnected contribution at vanishing momentum transfer divided by the corresponding connected contribution $F_{\mathrm{S}}^{\pi}(0)_{\text {disc }} / F_{\mathrm{S}}^{\pi}(0)_{\text {con }}$ plotted against the pion mass. Different colours denote different values of the lattice spacing. Except for the lightest ensemble (G8), which might simply be an outlier, we find no significant dependence on the pion mass. However, the relative contribution of the disconnected diagram has a very pronounced dependence on the lattice spacing. The smaller the lattice spacing, the smaller is the relative disconnected contribution $F_{\mathrm{S}}^{\pi}(0)_{\text {disc }} / F_{\mathrm{S}}^{\pi}(0)_{\text {con }}$ to the form factor.

\subsection{The scalar radius}

The scalar radius of the pion is determined from the $Q^{2}$ dependence of the form factor at vanishing momentum transfer. To estimate the scalar radius we use a linear parametrisation, i.e.

$$
F_{\mathrm{S}}^{\pi}\left(Q^{2}\right)=F_{\mathrm{s}}^{\pi}(0)\left(1-\frac{1}{6}\left\langle r^{2}\right\rangle_{\mathrm{s}}^{\pi} Q^{2}+\mathcal{O}\left(Q^{4}\right)\right) .
$$

To determine the scalar radius on a given ensemble we fit a function of the form (7) to our form factor results at the three different momentum transfers. Note, that the absolute normalization of the form factor is not known, since the multiplicative renormalisation $Z_{s}$ has not been determined. However, in the calculation of the scalar radius $Z_{s}$ drops out. For the lightest ensemble G8, the ratios for the third momentum transfer were too noisy to obtain a useful signal. Thus, we have to resort to match a linear function to two $Q^{2}$ values only for this ensemble. For all other ensembles where data for three different momentum transfers is available we find that the differences between using two or three momenta is not significant.
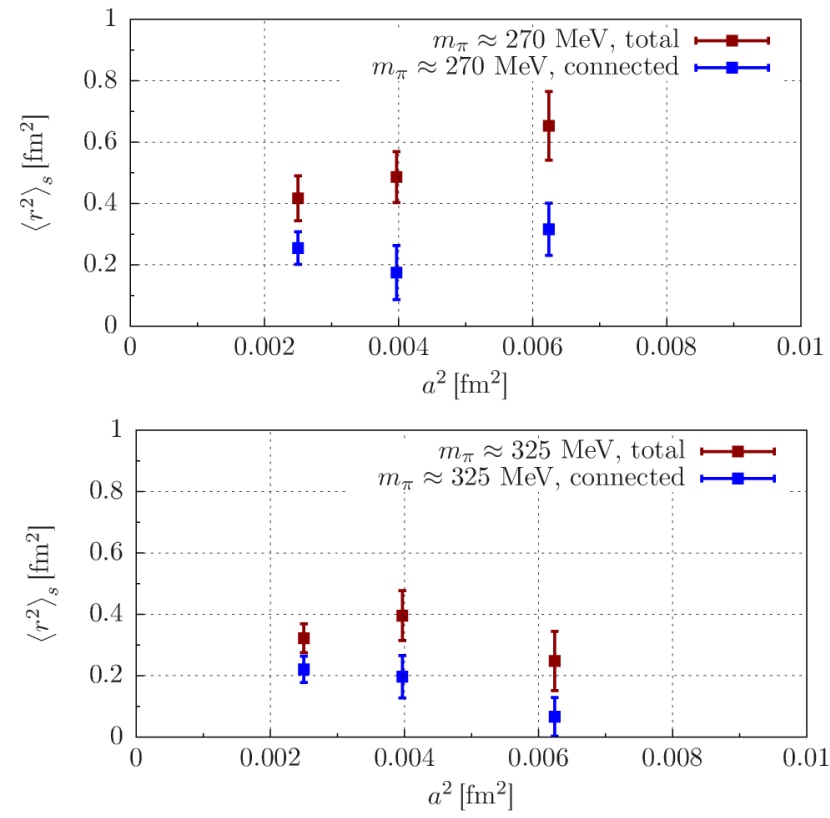

Fig. 3. The scalar radius at fixed pion mass plotted against the squared lattice spacing $a^{2}$. The upper panel shows results for a pion mass of $m_{\pi} \approx 270 \mathrm{MeV}$, the lower panel for $m_{\pi} \approx$ $325 \mathrm{MeV}$. Red and blue points denote results for the total and connected radius, respectively.

To investigate a possible dependence of the scalar radius on the lattice spacing we compare the results from ensembles with different lattice spacings at roughly the same pion mass. Within the CLS ensembles we have two sets of such ensembles (cf. table 1): $m_{\pi} \approx 270 \mathrm{MeV}$ (B6, F7, O7) and $m_{\pi} \approx 325 \mathrm{MeV}$ (A5, F6, N6). In fig. 3 our results for the scalar radius on these ensembles are plotted against the squared lattice spacing $a^{2}$. The upper panel shows results for a pion mass of $m_{\pi} \approx 270 \mathrm{MeV}$ the lower panel for $m_{\pi} \approx 325 \mathrm{MeV}$.

While no clear trend in the lattice spacing dependence of the results is apparent over the full range of lattice spacings studied, there seems to be a tendency for the full and connected results to approach each other at the smallest lattice spacings. In the following section we will therefore study cut-off effects both when in- or excluding the coarsest lattice spacing.

Although we do not find a significant dependence on the lattice spacing, we will include a term linear in $a^{2}$ for performing a combined extrapolation of the scalar radius to the physical point.

\section{Chiral and continuum extrapolation}

The use of gauge ensembles with different pion masses and lattice spacings allows for an extrapolation to the physical point $m_{\pi} \rightarrow m_{\pi \text {,phys }}$ and $a \rightarrow 0$. For the dependence on the pion mass we use the expression (4) from NLO $\chi \mathrm{PT}$. Since we use an $O(a)$-improved action and because no quark bilinears that could mix with $\bar{q} q$ at $O(a)$ exist, 


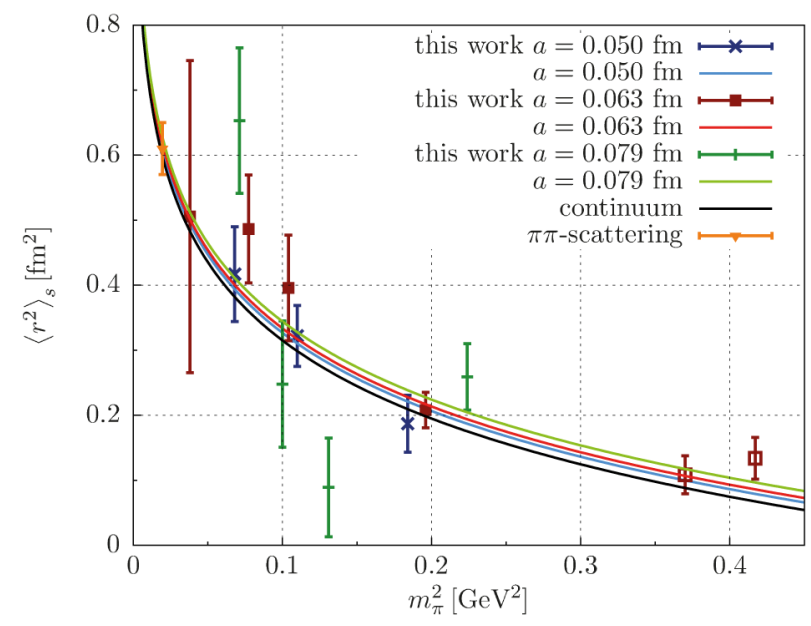

Fig. 4. Our results for the scalar radius plotted against the pion mass $m_{\pi}^{2}$ including the result from a combined chiral and continuum extrapolation. Different colours denote different lattice spacings. The black line is the pion mass dependence in the continuum from our combined fit. The two right most points (open symbols) at $m_{\pi}^{2} \approx 0.37 \mathrm{GeV}^{2}(\mathrm{E} 4)$ and $m_{\pi}^{2} \approx 0.42 \mathrm{GeV}^{2}$ (E3) are not included in the fit. The orange point at the physical pion mass shows the value from $\pi \pi$-scattering [1].

we expect lattice artefacts to be of order $a^{2}$ (up to contributions from gluonic operators, which we expect to be very small). Thus, we include a term $\propto a^{2}$ in a combined fit of the scalar radius for all gauge ensembles (cf. table 1 ):

$\left\langle r^{2}\right\rangle_{\mathrm{S}}^{\pi}=-\frac{1}{(4 \pi F)^{2}} \frac{13}{2}+\frac{6}{(4 \pi F)^{2}}\left[\bar{\ell}_{4}+\ln \left(\frac{m_{\pi, \mathrm{phys}}^{2}}{m_{\pi}^{2}}\right)\right]+b a^{2}$.

The fit function (8) has two fit parameters, the low-energy constant $\bar{\ell}_{4}$ and the coefficient $b$ in the term which depends on the lattice spacing. When fitting our data for the scalar radius to the NLO expression (8) we apply a cut in the pion mass by imposing $m_{\pi}<500 \mathrm{MeV}$, which excludes the ensembles E3 and E4.

Additionally, we have repeated the fit using all data points (i.e. no mass cut) and a more aggressive mass cut $m_{\pi}<335 \mathrm{MeV}$. We find that the fit result is quite insensitive to mass cuts, and the fits produce consistent results with an acceptable $\chi^{2} /$ dof $\lesssim 2$. The spread among the results when different mass cuts are applied is negligible compared to the statistical error. Thus we use the fit with $m_{\pi}<500 \mathrm{MeV}$ to quote a final result for the scalar radius.

The result of this combined fit to our data is shown in fig. 4, where the scalar radius is plotted against the squared pion mass. The green, red and blue curves describe the pion mass dependence at individual values of the lattice spacing, i.e. $a=0.079 \mathrm{fm}, a=0.063 \mathrm{fm}$ and $0.050 \mathrm{fm}$. Clearly, the lattice spacing dependence is very small; we find $b=4.7 \pm 12.5$ in our fit, indicating that a possible dependence on $a^{2}$ cannot be resolved with the current statistical accuracy of our data. To clarify whether the larger scatter of the results at the coarsest lattice spacing is responsible for this, we have also performed a fit to the finest two lattice spacings only, obtaining a result that is fully compatible with the fit to all three lattice spacings within statistical uncertainties. The same applies if we perform a fit without including cut-off effects by putting $b=0$ in eq. (8), indicating that cut-off effects are under control in our study.

The black line in fig. 4 shows the pion mass dependence in the continuum, $a=0$. At the physical pion mass we find for the scalar radius

$$
\left\langle r^{2}\right\rangle_{\mathrm{S}}^{\pi}=0.600 \pm 0.052 \mathrm{fm}^{2} .
$$

This is in excellent agreement with the value $0.61 \pm$ $0.04 \mathrm{fm}^{2}$ which was extracted from $\pi \pi$-scattering in [1] (cf. the orange point in fig. 4).

One has to note that the pion mass dependence of the scalar radius is completely driven by $\chi \mathrm{PT}$ (cf. eq. (4)), and the fit parameter $\bar{\ell}_{4}$ only changes the offset of the black curve in fig. 4 . We find that our results for the radius show the expected pion mass dependence from NLO $\chi \mathrm{PT}$, confirming its applicability.

From the combined fit we can extract the low-energy constant $\bar{\ell}_{4}$, which served as a fit parameter. We find

$$
\bar{\ell}_{4}=4.54 \pm 0.30
$$

which is in very good agreement with the value $\bar{\ell}_{4}=$ $4.62 \pm 0.22$ quoted in the current FLAG report [13] for the $N_{f}=2$ flavour theory.

In our previous work we found that the disconnected contribution to the scalar radius is important, which is in qualitative agreement with what has been found in partially quenched chiral perturbation theory [6]. We can confirm this using our new data by repeating the same combined fit for the scalar radius using the connected contribution only: we indeed find a smaller value for the scalar radius $\left\langle r^{2}\right\rangle_{\mathrm{s}}^{\pi, \text { conn }}=0.532 \pm 0.042 \mathrm{fm}^{2}$, corresponding to a smaller value for the low-energy constant $\bar{\ell}_{4}^{\text {conn }}=4.15 \pm 0.24$, which would disagree with the determination of $\bar{\ell}_{4}$ from other processes.

\section{Summary}

We have extended our previous study of the pion scalar radius using additional lattice spacings and pion masses in order to enable a fully controlled chiral and continuum extrapolation. We find that discretisation artefacts are mild, and that the pion mass dependence of the scalar radius is well described by NLO $\chi \mathrm{PT}$. From a combined extrapolation to the physical point, we are able to extract the low-energy constant $\bar{\ell}_{4}=4.54 \pm 0.30$ in good agreement with the FLAG [13] average.

The disconnected part of the pion three-point function contributes significantly to the slope of the scalar form factor near $Q^{2}=0$ and thus to the scalar radius of the pion. The inclusion of the disconnected diagrams is therefore essential in order to capture the correct physics. 
While our results do not include the contribution of the strange quark, these are expected to be suppressed by the mass of the strange quark and are unlikely to significantly impact the overall result for the radius.

We acknowledge useful discussions with Andreas Jüttner, Bastian Brandt and Harvey B. Meyer. Some of our calculations were performed on the "Wilson" HPC Cluster at the Institute for Nuclear Physics, University of Mainz. We thank Dalibor Djukanovic and Christian Seiwerth for technical support. We are grateful for computer time allocated to project HMZ21 on the BlueGene computers "JUGENE" and "JUQUEEN" at NIC, Jülich. This research has been supported in part by the DFG in the SFB 1044. We are grateful to our colleagues in the CLS initiative for sharing ensembles.

Open Access This is an open access article distributed under the terms of the Creative Commons Attribution License (http://creativecommons.org/licenses/by/4.0), which permits unrestricted use, distribution, and reproduction in any medium, provided the original work is properly cited.

\section{References}

1. G. Colangelo, J. Gasser, H. Leutwyler, Nucl. Phys. B 603, 125 (2001) hep-ph/0103088.

2. J. Gasser, H. Leutwyler, Ann. Phys. 158, 142 (1984).

3. J. Gasser, H. Leutwyler, Phys. Lett. B 125, 325 (1983).

4. Particle Data Group Collaboration (K. Olive et al.), Chin. Phys. C 38, 090001 (2014).

5. J. Bijnens, G. Ecker, Annu. Rev. Nucl. Part. Sci. 64, 149 (2014) arXiv:1405.6488.

6. A. Jüttner, JHEP 01, 007 (2012) arXiv:1110.4859.

7. V. Gülpers, G. von Hippel, H. Wittig, Phys. Rev. D 89, 094503 (2014) arXiv:1309.2104.

8. S. Capitani, M. Della Morte, G. von Hippel, B. Knippschild, H. Wittig, PoS LATTICE2011, 145 (2011) arXiv:1110.6365.

9. P.A. Boyle, J.M. Flynn, A. Jüttner, C.T. Sachrajda, J.M. Zanotti, JHEP 05, 016 (2007) hep-lat/0703005.

10. S. Güsken et al., Phys. Lett. B 227, 266 (1989).

11. C. Alexandrou, F. Jegerlehner, S. Güsken, K. Schilling, R. Sommer, Phys. Lett. B 256, 60 (1991).

12. UKQCD Collaboration (C. Allton et al.), Phys. Rev. D 47, 5128 (1993) hep-lat/9303009.

13. S. Aoki et al., Eur. Phys. J. C 74, 2890 (2014) arXiv:1310.8555. 